\title{
Interdisciplinary Anesthesia Tray Revision Project: Reducing the Opportunity for Human Error
}

\author{
Smith $K L^{1^{*}}$, Sharp $C^{2}$, Smith $E^{1}$, Currie $M^{1}$, Hall $K P^{1}, V u T^{1}$, Lee $M^{1}$ and Cooper $R L^{3}$ \\ ${ }^{1}$ Department of Pharmacy, Regional One Health, USA \\ ${ }^{2}$ Department of Anesthesiology, University of Tennessee Health Science Center, USA \\ ${ }^{3}$ Department of Anesthesiology, University of Wisconsin, USA
}

*Corresponding author: Smith KL, Department of Pharmacy, Regional One Health, 877 Jefferson Ave, Memphis, TN 38103, E-mail: kyork@regionalonehealth.org

\begin{abstract}
Background: Medication errors cause an estimated 100,000 to 400,000 patient deaths in the United States annually (IOM). Previous reviews on this alarming statistic consistently identify human error as the most common etiology. The potential for medication errors is especially high risk in the operating suite, where the provider simultaneously selects, doses and verifies medications to be administered to patients. Additionally, a lack of standardization and error reduction strategies in this setting creates the environment for medication administration errors, prompting multiple recommendations for interventions to minimize human error.
\end{abstract}

Methods: This project took place in three phases. In the Evaluation phase, evidence-based literature on anesthesia medication safety practices were evaluated, and high-risk/ look-alike, sound-alike medications on anesthesia medication trays were identified. In the Standardization phase, the interdisciplinary team developed strategies to organize the medication trays to improve safety. These standardized strategies were executed throughout the anesthesia mediation trays in the final Implementation phase.

Results: A total of eight-three changes were made to the anesthesia medication trays. Sixty medications were removed, with an average of ten removals per tray. High risk medications were replaced with unit-of-use or ready-to-use syringes when possible, with an average of four replacements per tray. The greatest number of medication tray changes was in the trauma operating suite with a total of twenty-eight.

Conclusions: Numerous opportunities were identified to decrease the potential for human error by standardizing anesthesia medication trays with a focus on optimizing medication safety. Organization and standardization of anesthesia medication trays is an economical strategy to decrease opportunity for human error in the operating suite that requires minimal capital outlay. Although previous studies have recommended strategies and technologies to improve medication safety in the operating suites; these interventions have yet to be implemented. Our unique study demonstrates successful intervention implementation.

\section{Keywords}

Anesthesiology, Medication error, Operating room

\section{Introduction}

Medication safety technologies commonly implemented throughout health systems to decrease medication errors are often not practical or available in anesthesia practice or the operating suites due to the unique environment. For example, medication safety strategies, such as barcoding and pharmacist verification, are not widely available at the point of care. Providers in the operating rooms are simultaneously selecting (point of care dispensing, usually from drug trays), dosing, preparing or compounding, and administering medications without nurse, patient, pharmacist, or other double-check or verification. This leaves the single provider environment at an especially high risk for medication errors.

In 1999, The Institute of Medicine (IOM) reported that from 44,000 to 98,000 patients die from medication-related errors each year [1]. Cooper, et al. found the anesthesia medication error rate of 1:203 anesthetics, with the most common errors being wrong dose and wrong drug (syringe swap); both caused by human error [2]. In 
Table 1: Breakdown of these eighty-three interventions by removals, replacements, additions and location.

\begin{tabular}{|l|l|l|l|l|}
\hline Location & Removals & Replacements & Additions & Total Changes \\
\hline Trauma OR & 20 & 5 & 3 & 28 \\
\hline Burn OR & 12 & 4 & 4 & 20 \\
\hline Outpatient OR & 14 & 4 & 0 & 18 \\
\hline Inpatient OR & 14 & 3 & 0 & 17 \\
\hline
\end{tabular}

the seven years following the IOM report, greater than 11,200 perioperative medication errors were reported to the international medication error reporting database, MEDMARX [3]. This finding led the Anesthesia Patient Safety Foundation (APSF) to create many medication safety recommendations including medication standardization to reduce the amount of anesthesia medication errors. To our knowledge, few studies in the English literature describe the reconfiguration of anesthesia trays as an intervention strategy to decrease the potential for anesthesia medication errors, focusing on medication safety. The purpose of this quality assurance project was to standardize anesthesia drug trays that incorporated evidence-based interventions designed to decrease opportunity for human error and to report a common sense and low-cost approach to implementing those strategies.

\section{Methods}

This project took place in three separate phases. Phase I was the Evaluation Phase. In Phase I, evidence-based literature was evaluated regarding anesthesia medication practices. Led by the Chief of Anesthesiology, this phase identified high risk, problem-prone medications on the anesthesia trays. This evaluation included medications on the anesthesia trays provided to four operating suites throughout the organization, trauma, burn, outpatient, and inpatient elective. This was an interdisciplinary review with anesthesia and pharmacy. A project timeline was developed to reconfigure all 36 anesthesia trays. The project began in February 2016 and continued for three months through May 2016.

During Phase I, medication tray contents were reviewed by pharmacologic category, quantity/par level and frequency of use. Also identified were potential look alike/sound alike (LASA) medications, commercially available premixed medications, and unnecessary high alert medications. Visual, color coding similarities were considered. Trays were sequentially evaluated from each operating suite.

Phase II was the Standardization Phase. During Phase II, the interdisciplinary team determined the optimal contents of the tray, with a focus on the previously identified medication safety enhancements. Strategies commonly utilized were distancing of visually similar products and LASA medications, establishing inventory quantities based on utilization and ordering pre-mixed doses when available. Unnecessary high-risk medications were identified for removal. Medications not essential to anesthesia care were either removed entirely or moved to the automated dispensing cabinet.
The final phase, Phase III, was the implementation phase. Phase II findings were implemented, and the process of reconfiguring anesthesia medication trays was undertaken. With minor exceptions, contents were standardized and identical for all locations. Medications were barcoded into distinctive and separate compartments in the trays. The pharmacy prepared and sealed trays with verified medications. Pharmacy personnel delivered and exchanged trays daily. Any request for a change required approval by the Chair of Anesthesiology. All thirty-six trays were completed by May 2016.

\section{Results}

A total of eighty-three changes were made to the anesthesia medication trays during the implementation phase. The most common intervention made was the removal of medications, with a total of sixty medications removed. This was followed by replacements and additions at sixteen and seven, respectively. Common actions taken included removal of medications requiring mixing during procedure, high-risk medications, medications available in Automated Medication Dispensing Cabinet, and medications rarely used. After evaluation of medication safety implications, rationale for adding medications to the medication tray included medications previously needed but unavailable. Highrisk medications were replaced with unit-of-use when possible. Table 1 demonstrates the breakdown of these eighty-three interventions by removals, replacements, additions and location.

There was an average of ten unique medication removals per tray. High risk medications were removed if unnecessary or replaced by ready to use dosage forms. If LASA or visually similar medications could not be removed, they were strategically placed in distant locations on the tray. An average of four unique medication replacements were made to incorporate more pre-filled syringes. High risk medications were replaced with unitof-use or ready-to-use syringes when possible, with an average of two unique medication replacements per tray. An average of three unique medications were added to each tray that were previously needed, but unavailable. Figure 1 depicts the details of these changes. The greatest number of medication tray changes were made in the trauma operating suite with a total of twenty-eight, followed by burn operating suite with twenty changes.

\section{Discussion}

Anesthesia medication errors are poorly defined, 


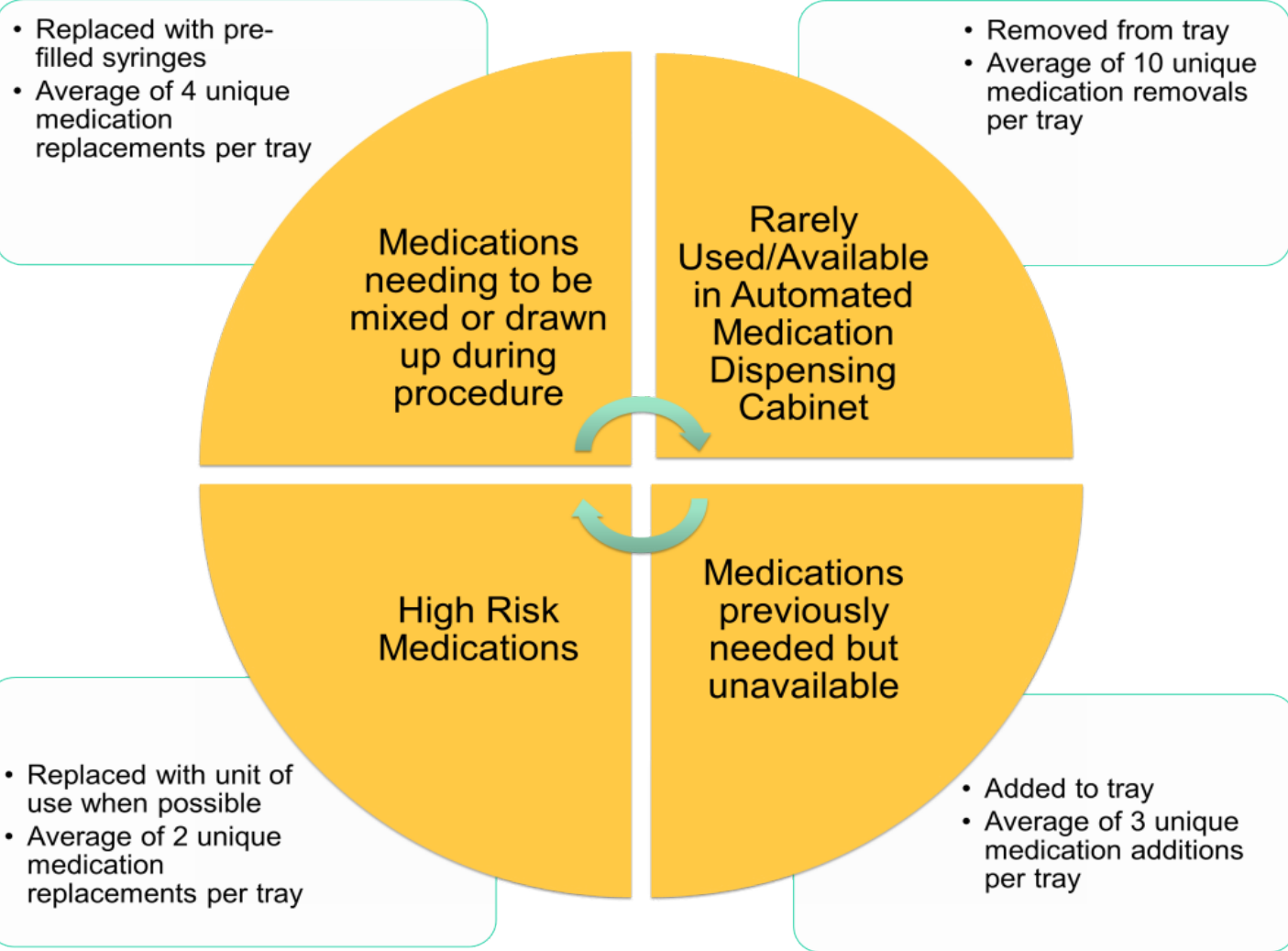

Figure 1: Depicts the details.

underreported, and are recently being recognized with increasing frequency. Multiple studies have identified human error as the most common cause of anesthesia medication error, due to the lack of standardization of medications, absence of greater than single provider verification, and lack of safety technologies such as barcoding $[2,4]$. In response to multiple reports of anesthesia medication errors, Jensen, et al. conducted the first systematic review of the literature on reducing intravenous medication errors in anesthesia in 2004. Recommendations to avoid error in drug administration included standardization of labeling and packaging, organization of medication trays, and ready-to-use medications [5]. A more recent study showed a reduction in error rate when these intervention strategies were implemented [6].

In response to these challenges, the APSF summit, held in 2010, recommended development of new strategies to decrease medication errors in anesthesia. Recommendations included the need for medication standardization, high-alert medications only available in standardized concentrations and ready-to-use preparations, standardized labels, and new technology [7]. Like all medication error reviews, identification and rates of errors vary according to definition and methodology, including inconsistencies with handling of near-miss events. Secondary to these shortcomings, it is well recognized that many anesthesia medication errors go unrecognized and unreported [8]. While concerning, this challenge is certainly not unique to anesthesia practice.
Previously reported MEDMARX data on the number of perioperative medication errors have their own limitations, including collection of data mostly from small community hospitals, few academic centers with training programs, reporting by pharmacists (not anesthesiologists), and the fact that anesthesia-specific errors were not addressed at all, suggesting an even higher rate of medication errors in perioperative settings.

Despite previous recommendations to improve intraoperative medication safety, Common errors described included administering the wrong medication, or calculating and administering the wrong dose [9]. Several other studies have shown similar anesthesia medication errors rates to occur around the world in various anesthesia settings [10-13]. In addition, a recent study by Nanji found a significantly higher medication error rate in anesthesia when providers were observed versus when they voluntarily reported errors [14]. The authors reported anesthesia medication errors to occur in approximately one in twenty doses administered (as opposed to number of anesthetics, as reported in previous studies). Under-reporting was found with voluntary reporting systems by both Cohen and Cullen, et al. as early as $2000[15,16]$. To our knowledge, only two studies in the English literature describe the reconfiguration of anesthesia trays as an intervention strategy to decrease the potential for anesthesia medication errors, focusing on medication safety $[5,6]$. In seven years since the APSF recommendations, few studies describe implementation of other suggested anesthesia safety interventions. Nanji's study establishes a concerning 
error rate that has not decreased. This study suggests that the observed anesthesia medication error rate may even be higher than previously recognized by voluntary reporting [14].

In 2017, Wahr, et al. conducted a comprehensive literature review and found 138 specific recommendations to minimize error in the operating suites. Despite identifying a commonality in the literature for needed interventions, this study noted the lack of implementation of recommendations to improve anesthesia medication safety [9]. Similar to previous reviews, strategies to improve medication safety included medication standardization, drug labeling, and limitation of high risk medications, among others. Secondary to the increased error rate shown by Nanji and colleagues, Kitson, et al. presented a conceptual framework regarding imple- mentation of evidence-based practices, which can shed light on why implementation often falters [17]. A key component of this study was recognition of a departmental and organizational culture of safety as an essential component for success.

Organization of anesthesia medication trays is relatively simple and does not require a large capital outlay. The primary cost of the improvement is labor associated with reconfiguring the trays and barcoding the medications for verification by location in the tray. Knowledge of anesthesia medication safety challenges is essential for successful anesthesia medication tray reconfiguration, along with a strong interdisciplinary team and leadership committed to medication safety. The missing link of technology to close the loop on systematic prevention of anesthesia medication errors is a lack

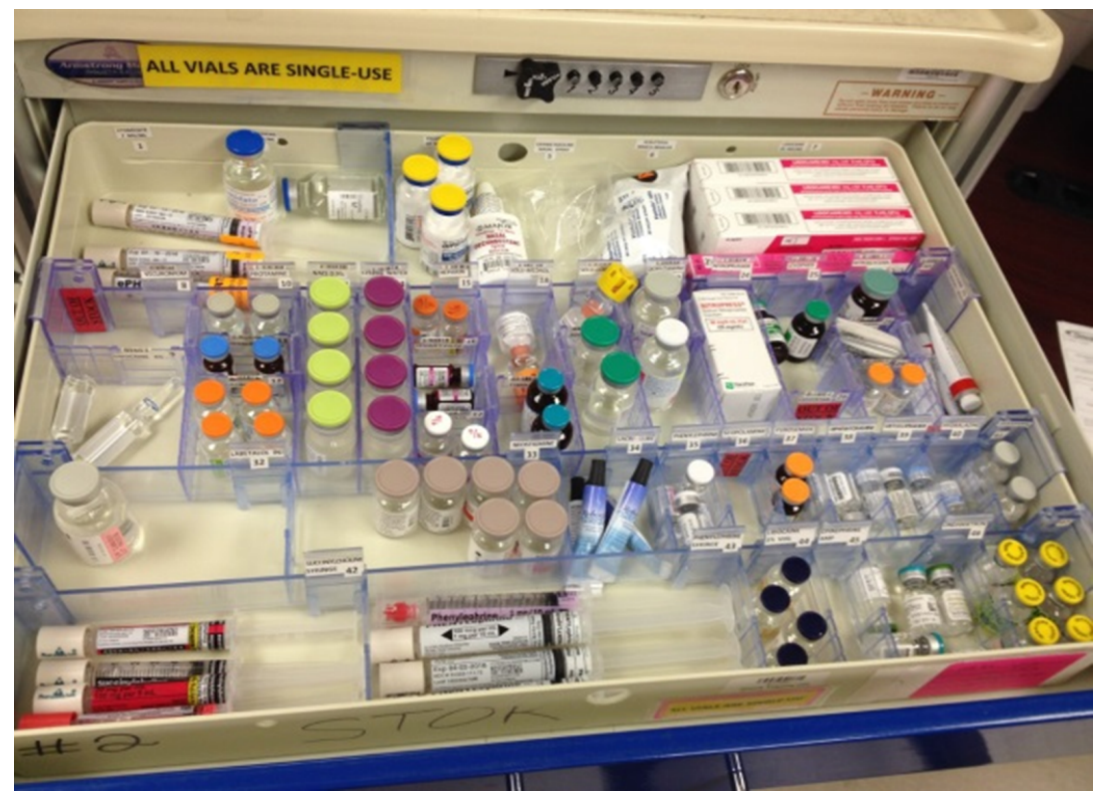

Before

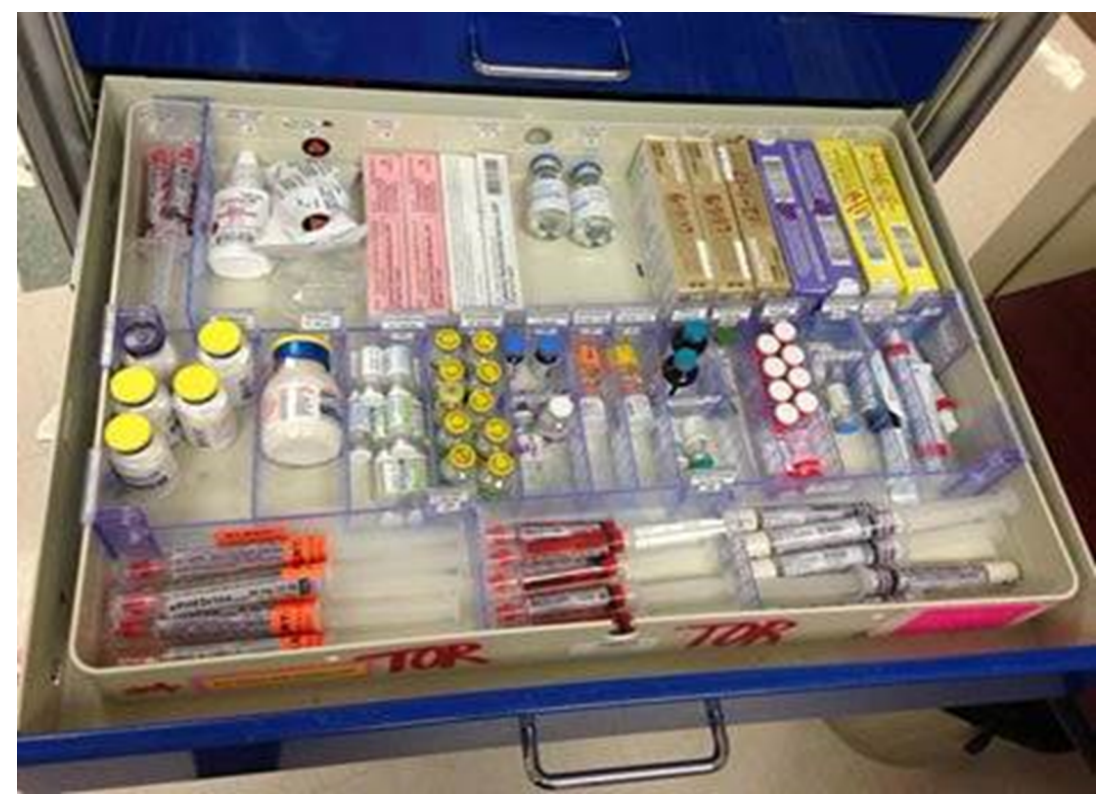

After

Figure 2: Before and after results. 
of available barcoding at the point of administration to provide electronic verification to the provider of correct drug, dose, allergies, or drug-drug interactions. To date, no commercially available technology exists in the US to achieve this needed intervention strategy to reduce medication errors.

Multiple opportunities were identified at our institution by our interdisciplinary project team to enhance anesthesia medication safety in the operating suite. Anesthesia medication trays were reorganized and configured to decrease the opportunity for human error. Figure 2 demonstrates the before and after results of our changes made. By decreasing the amount of medications in each tray, previously cluttered trays became more organized. If the medication was rarely used, or if it was available in the automated medication dispensing cabinet, it was removed. With fewer medications to choose from each in each tray, there were fewer opportunities for the practitioners to choose the wrong drug. Pre-filled syringes replaced ready-mix ampules and vials; therefore, leading to fewer opportunities to prepare the wrong dose or inadvertently administer undiluted medications. Lack of standardization and presence of LASA and unnecessary high-risk medications represent a system fallacy that can set a practitioner up to unintentionally commit an error. It is essential that system errors be addressed to prevent human errors.

It is well established that medications should be selected by careful examination of the label [5]. Unfortunately, temptation exists to circumvent this expectation by selecting medications based on similar packaging and color, and the very nature of anesthesia practice in stressful environments with considerable multi-tasking occurring simultaneously sets practitioners up for failure. This can be particularly problematic in an era of drug shortages when vial or ampule appearances may change unexpectedly based on manufacturer availability. When this occurs, these medications should be strategically placed in distant locations to minimize opportunity for this error. Since drug shortages are frequent occurrences, this activity should occur on an ongoing basis when products from different manufacturers are added to the tray.

Our experience during this anesthesia medication safety intervention clearly demonstrates the need for strong leadership to maintain appropriate changes and the ability to establish and nurture solid interdisciplinary relationships. This is required to diffuse many practitioner preference-based requests that occur, especially in the initial two weeks following standardization. A strong relationship between the anesthesia team and pharmacy will likely result in the minimization of medication errors. Also critical to the success of the intervention is knowledge in evidence-based anesthesia medication safety issues and recommendations. Without this expertise, there is the risk that standardization will be based on individual practitioner preferences instead of validated safety initiatives.

There are several limitations to this report. First, the design was not to show an absolute reduction in anesthesia medication error rate, but to standardize the anesthesia drug trays to achieve evidence-based recommendations that have been shown to reduce those errors. Second, no post-implementation analysis of anesthesia medication error rate was undertaken, but standardization has been shown to reduce errors. Third, these interventions are not the only ones that have been shown to reduce errors, and there may be alternate approaches that health systems may consider that achieve similar results. Finally, we chose not to include the specific medications removed, replaced, or moved, as each institution may have different definitions to what is considered high-risk or have different medication needs than what we can recommend.

\section{Conclusion}

With increasing frequency, technologies are being introduced to enhance medication safety both in and out of the operating suite. Many of these new technologies require substantial capital investment that prevents their application in every organization. Other than labor costs, this operating suite safety intervention uses contemporary safety principles to reconfigure anesthesia trays to enhance safety, requires minimal financial expenditures, and is easily incorporated in every organization throughout the United States. Practitioners should readily embrace this simplistic, yet critical, intervention to enhance the unique safety environment in the operating suite, because our patients demand the benefits it offers.

\section{References}

1. Homested L (2000) Institute of Medicine report: To err is human: Building a safer health care system. Fla Nurse 48: 6.

2. Cooper L, DiGiovanni N, Schultz L, Taylor AM, Nossaman B (2012) Influences observed on incidence and reporting of medication errors in anesthesia. Can J Anaesth 59: 562570.

3. (2007) MEDMARX Data Report. United States Pharmacopeia.

4. Craig J, Wilson ME (1981) A survey of anaesthetic misadventures. Anaesthesia 36: 933-936.

5. Jensen LS, Merry AF, Webster CS, Weller J, Larsson L (2004) Evidence-based strategies for preventing drug administration errors during anaesthesia. Anaesthesia 59: 493-504.

6. Merry AF, Craig S Webster, Jacqueline Hannam (2011) Multimodal system designed to reduce errors in recording and administration of drugs in anaesthesia: Prospective randomized clinical evaluation. BMJ 343: 5543.

7. Eichhorn JH (2010) Medication safety in the operating room: Time for a new paradigm. APSF Summit Conference Proceedings, APSF Newsletter 25: 1-20.

8. Cooper L, Nossaman B (2013) Medication errors in anes- 
thesia: A review. Int Anesthesiol Clin 51: 1-12.

9. Wahr JA, Abernathy JH, Lazarra EH, Keebler JR, Wall MH, et al. (2016) Medication safety in the operating room: Literature and expert-based recommendations. $\mathrm{Br} \mathrm{J}$ Anaesth 118: $32-43$.

10. Webster CS, Merry AF, Larsson L, McGrath KA, Weller J (2001) The frequency and nature of drug administration error during anaesthesia. Anaesth Intensive Care 29: 494500.

11. Llewellyn RL, Gordon PC, Wheatcroft $D$, Lines $D$, Reed A, et al. (2009) Drug administration errors: a prospective survey from three South African teaching hospitals. Anaesth Intensive Care 37: 93-98.

12. Kahn FA, Hoda MQ (2001) A prospective survey of intra-operative critical incidents in a teaching hospital in a developing country. Anaesthesia 56: 177-182.
13. Yakamoto M, Ishikawa S, Makita K (2008) Medication errors in anesthesia: an 8-year retrospective analysis at an urban university hospital. J Anesth 22: 248-252.

14. Nanji KC, Patel A, Shaikh S, Seger DL, Bates DW (2016) Evaluation of perioperative medication errors and adverse drug events. Anesthesiology 124: 24-34.

15. Cohen M (2000) Why error reporting systems should be voluntary. Institute of safe medication practices. BMJ 320 : 728-729.

16. Cullen D, Bates D, Leape $L$ (2000) Prevention of adverse drug events: A decade of progress in patient safety. Journal of Clinical Anesthesia 12: 600-614.

17. Kitson A, Harvey G, McCormack B (1998) Enabling the implementation of evidence-based practice: A conceptual framework. Health Care 7: 149-158. 